Article

\title{
Clinical Characteristics of COVID-19 Patients and Application to an Artificial Intelligence System for Disease Surveillance
}

\author{
Ying-Chuan Wang ${ }^{1,+} \mathbb{D}$, Dung-Jang Tsai ${ }^{2,3,+}{ }^{\mathbb{D}}$, Li-Chen Yen ${ }^{4} \mathbb{D}$, Ya-Hsin Yao ${ }^{5}$, Tsung-Ta Chiang ${ }^{6}$, \\ Chun-Hsiang Chiu ${ }^{6, *}$, Te-Yu Lin ${ }^{6}$, Kuo-Ming Yeh ${ }^{6}$ and Feng-Yee Chang ${ }^{6}$
}

check for

updates

Citation: Wang, Y.-C.; Tsai, D.-J.;

Yen, L.-C.; Yao, Y.-H.; Chiang, T.-T.;

Chiu, C.-H.; Lin, T.-Y.; Yeh, K.-M.;

Chang, F.-Y. Clinical Characteristics

of COVID-19 Patients and

Application to an Artificial

Intelligence System for Disease

Surveillance. J. Clin. Med. 2022, 11,

1437. https://doi.org/10.3390/

jcm11051437

Academic Editor: Danilo Buonsenso

Received: 18 January 2022

Accepted: 3 March 2022

Published: 5 March 2022

Publisher's Note: MDPI stays neutral with regard to jurisdictional claims in published maps and institutional affiliations.

Copyright: (C) 2022 by the authors. Licensee MDPI, Basel, Switzerland. This article is an open access article distributed under the terms and conditions of the Creative Commons Attribution (CC BY) license (https:// creativecommons.org/licenses/by/ $4.0 /)$
1 Department of Family Medicine, Tri-Service General Hospital, National Defense Medical Center, Taipei 11499, Taiwan; popoga96@gmail.com

2 Graduate Institute of Life Sciences, National Defense Medical Center, Taipei 11499, Taiwan; oo800217@gmail.com

3 School of Public Health, National Defense Medical Center, Taipei 11499, Taiwan

4 Department of Microbiology and Immunology, National Defense Medical Center, Taipei 11499, Taiwan; yenlichen1030@gmail.com

5 National Defense Medical Center, Taipei 11499, Taiwan; yamcom13579@gmail.com

6 Division of Infectious Diseases and Tropical Medicine, Department of Internal Medicine, Tri-Service General Hospital, National Defense Medical Center, Taipei 11499, Taiwan; cgdada@gmail.com (T.-T.C.); lin.deyu@msa.hinet.net (T.-Y.L.); kmyeh@mail.ndmctsgh.edu.tw (K.-M.Y.); fychang@mail.ndmctsgh.edu.tw (F.-Y.C.)

* Correspondence: pipi10279@gmail.com; Tel.: +886-2-87927257

+ These authors contributed equally to this work.

\begin{abstract}
During the coronavirus disease (COVID-19) pandemic, we admitted suspected or confirmed COVID-19 patients to our isolation wards between 2 March 2020 and 4 May 2020, following a welldesigned and efficient assessment protocol. We included 217 patients suspected of COVID-19, of which 27 had confirmed COVID-19. The clinical characteristics of these patients were used to train artificial intelligence (AI) models such as support vector machine (SVM), decision tree, random forest, and artificial neural network for diagnosing COVID-19. When analyzing the performance of the models, SVM showed the highest sensitivity (SVM vs. decision tree vs. random forest vs. artificial neural network: $100 \%$ vs. $42.86 \%$ vs. $28.57 \%$ vs. $71.43 \%$ ), while decision tree and random forest had the highest specificity (SVM vs. decision tree vs. random forest vs. artificial neural network: $88.37 \%$ vs. $100 \%$ vs. $100 \%$ vs. $94.74 \%$ ) in the diagnosis of COVID-19. With the aid of AI models, physicians may identify COVID-19 patients earlier, even with few baseline data available, and segregate infected patients earlier to avoid hospital cluster infections and to ensure the safety of medical professionals and ordinary patients in the hospital.
\end{abstract}

Keywords: COVID-19; artificial intelligence; support vector machine (SVM); decision tree; random forest; artificial neural network

\section{Introduction}

Coronavirus disease 2019 (COVID-19) is an ongoing global pandemic caused by the severe acute respiratory syndrome coronavirus 2 (SARS-CoV-2). The clinical spectrum of COVID-19 appears to be broad, ranging from no symptoms to mild upper respiratory tract illness, severe pneumonia with respiratory failure, and death. The existence of asymptomatic patients and patients with non-specific symptoms may significantly delay the diagnosis of COVID-19 and present a serious threat to public health. The rising incidence and massive casualties of COVID-19 exert significant pressure on limited healthcare resources. The early diagnosis of asymptomatic or mild COVID-19 patients is essential to prevent the spread of the infection during the pandemic. However, the gold standard for COVID-19 diagnosis, the reverse transcriptase polymerase chain reaction (RT-PCR), takes a maximum of up to two days to give the result. There has also been a heavy shortage of 
RT-PCR test kits in many countries during the pandemic. Thus, effective tools are really needed to simplify the diagnosis and surveillance of COVID-19. Recently, researchers found that well-trained artificial intelligence (AI) can ensure accurate and rapid diagnosis or assist physicians to reduce manual labor. Some of these studies were conducted for AI-assisted COVID-19 diagnosis [1-18], some were conducted for predicting the prognosis of patients [19-28], and others were conducted for predicting the epidemic trend of COVID-19 [29-31].

Taiwan was initially expected to be one of the most affected countries owing to its geographic proximity and close people-to-people exchanges with China [32]. However, as the disease continues to spread globally, Taiwan has been able to contain the pandemic and minimize its impact on the daily lives of its citizens. Since the first confirmed case in Taiwan on 21 February 2020 [33], less than 1100 cases were reported in Taiwan till 10 April 2021. This is attributed to the government's rapid action including border control from the air and sea, adequate screening, quarantine of suspicious cases, identification of travelers infection risks, and comprehensive contact tracing. To coordinate the pandemic-prevention policies, our hospital set up two isolation wards to admit confirmed COVID-19 patients and suspected cases since 2 March 2020. Until 4 May 2020, 217 patients had been hospitalized into our isolation ward, of which 27 had a confirmed COVID-19 diagnosis. To facilitate diagnosing COVID-19, we tried to apply the clinical characteristics of these patients to different AI models and find the most effective one.

There are limited data on the clinical characteristics of COVID-19 patients in Taiwan. We aimed to delineate the epidemic prevention experience of our hospital under the guidance of Taiwan's government, clarify the differences in clinical characteristics between confirmed cases and COVID-19-negative patients admitted to our hospital, and apply the clinical characteristics to AI models for diagnosing COVID-19.

\section{Materials and Methods}

\subsection{Study Population}

Our study included adult patients (age $\geq 20$ years) with suspected or confirmed COVID-19 diagnosis, who were admitted to our isolation wards between 2 March 2020 and 4 May 2020. All patients with COVID-19 were confirmed by using real-time reversetranscriptase polymerase chain reaction (RT-PCR) assays from oropharyngeal swab specimens. The patients included travelers entering Taiwan with a positive COVID-19 test performed at the airport, symptomatic patients with a contact or travel history who visited our emergency room and needed hospitalization, and people with close contact with confirmed cases and who needed hospitalization. The study was approved by the Institutional Review Board of Tri Service General Hospital, and informed consent was obtained from all patients.

\subsection{COVID-19 Screening, Hospitalization, and Home Quarantine}

Figure 1 shows a flow diagram of the protocols followed for COVID-19 screening, hospitalization, and home quarantine. All travelers entering Taiwan were required to stay at home or at a quarantine hotel and undergo home quarantine for two weeks. Among them, individuals with symptoms were tested at the airport for SARS-CoV-2 viral nucleic acid using RT-PCR assays from oropharyngeal swab specimens. They had to stay at home or at the quarantine hotel while awaiting the test results. Individuals with a positive result in the COVID-19 test were sent to the appointed hospital for isolation and treatment. People with a negative result continued the two-week home quarantine. Symptomatic patients who visited our hospital with a travel or contact history were referred to our emergency room (ER) and tested for COVID-19. Patients who needed hospitalization were admitted to our isolation ward while waiting for the test result. Among them, patients with a positive result remained hospitalized in the isolation ward, while patients with a negative result were transferred to an ordinary ward. Patients in the ER who did not need hospitalization were asked to stay at home until the test results. Among them, patients 
with a positive result were admitted to the isolation ward, while patients with a negative result home-quarantined for two weeks. Individuals with close contact with confirmed cases were sent to the appointed hospital for COVID-19 testing, and symptomatic people among them were isolated in the dedicated ward until the results arrived. People without symptoms stayed at home or at a quarantine hotel while waiting for the result. Among them, people with positive results were arranged hospitalization in the isolation ward, while COVID-19-negative patients continued the home quarantine for two weeks. All transportation between the airport, home, the quarantine hotel, and the hospital were made through appointed cars instead of public transportation.

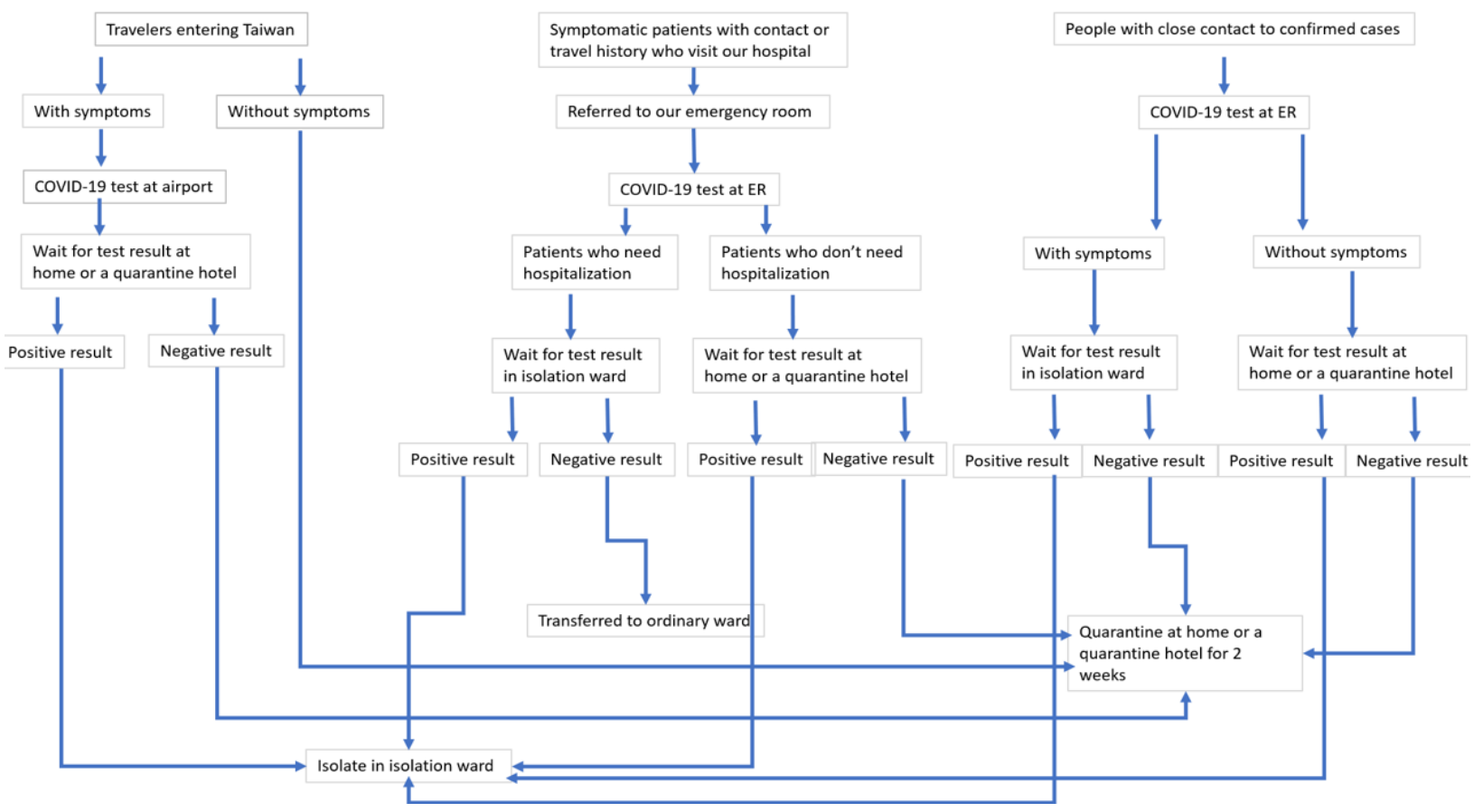

Figure 1. Flow diagram of COVID-19 screening, hospitalization, and home quarantine for travelers entering Taiwan, symptomatic patients who visited our emergency room, and people with close contact with confirmed cases.

\subsection{Obtaining the Demographic Data, Clinical Symptoms, and Laboratory Data}

Relevant clinical data of the enrolled people, including age, gender, underlying diseases, clinical symptoms, and laboratory data, were recorded. Laboratory data including white blood cell count, platelet count, neutrophil-to-lymphocyte ratio, renal function, liver function, levels of total bilirubin, C-reactive protein, D-dimer, and procalcitonin were examined and noted within $24 \mathrm{~h}$ after admission. Patients were diagnosed with pneumonia based on the lower respiratory tract symptom of cough, the systemic symptom of fever, and new onset radiology findings of infiltration [34,35].

\subsection{Statistical Analysis}

The patients were sub-grouped in confirmed COVID-19 patients and suspected cases with a negative result to compare their clinical characteristics, including demographic data, underlying diseases, symptoms, and laboratory data. All results were analyzed using a commercially available software package (SPSS, version 21.0; SPSS Inc., Chicago, IL, USA). Categorical variables were analyzed using the chi-square test, while continuous variables with categorical variables were analyzed with the independent two-samples $t$ test for comparison. All $p$-values were 2-tailed, and $p$-values of less than 0.05 were considered to indicate statistical significance. 


\subsection{Applying the Clinical Characteristics and Routine Laboratory Data to Train AI Models}

In order to obtain a confirmed COVID-19 predictive model ("Outcome" feature), we established four AI models including support vector machine (SVM), decision tree, random forest, and artificial neural network by inputting the above information comprising clinical characteristics (sex, age, temperature, SBP, DBP, PR, RR, fever, cough, headache, muscle ache, distorted sense of taste, distorted sense of smell, rhinorrhea, sore throat, chest tightness, dyspnea, diarrhea, eye illness, nausea and vomiting) and routine laboratory data (WBC $/ 1000, \mathrm{PLT}, \mathrm{Neu}(\%), \mathrm{ANC}, \mathrm{Lym}(\%), \mathrm{ALC}, \mathrm{Cr}, \mathrm{CRP}, \mathrm{AST}, \mathrm{ALT})$. We created training and testing sets by splitting the sample randomly to assess the performance of the model. A classifier can only be trained using retrospective data in the real world, and it will be used to classify future data. The machine learning construction process was to split all data into training and test datasets using $80 \%$ and $20 \%$ of the data. The process is shown in Figure 2 .

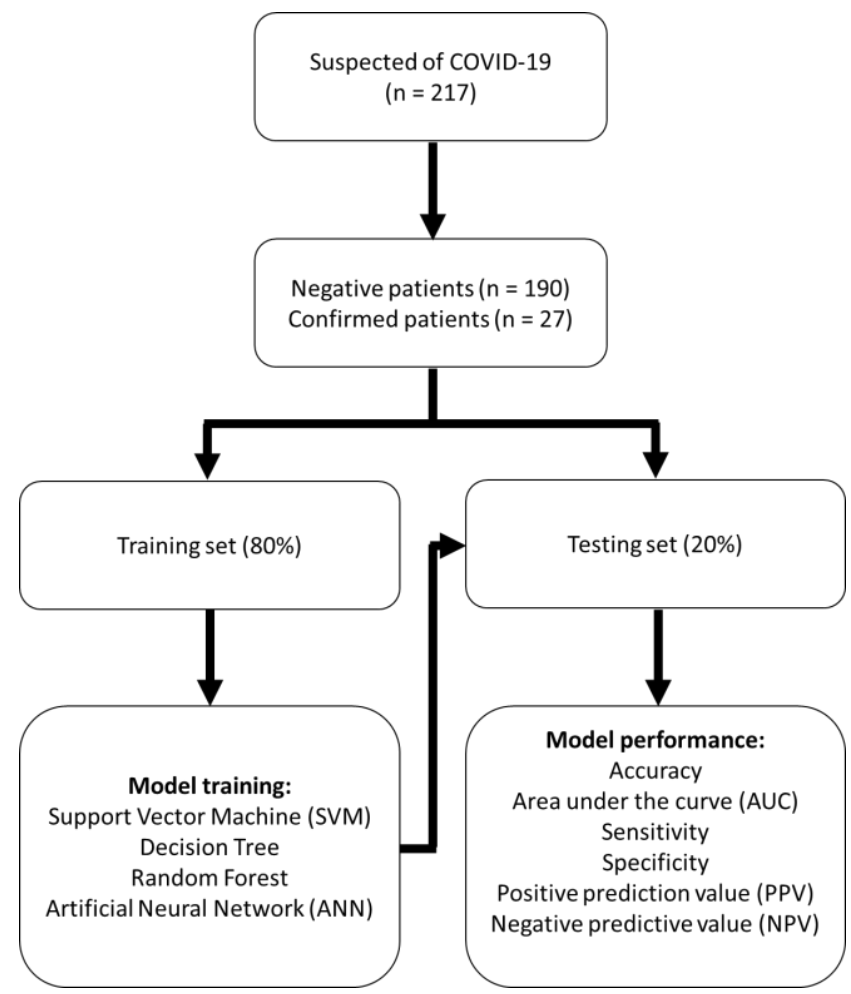

Figure 2. Flow chart of patients included in the model building and validation process.

\subsubsection{Support Vector Machines}

Support vector machines (SVMs) are common classifiers in machine learning. They map all samples to a hyperplane and separate them with a clear space. In addition, core tips are used to extend this hyperplane. SVMs have been shown to perform better in classifying free-text medical literature than naive Bayesian classifiers, $\mathrm{C} 4.5$ decision trees, and adaptive amplification [36]. In this study, we used the four most common kernel tips: linear, polynomial (degree $=3)$, radial basis, and sigmoid. We used the e1071 package $(R$ package version 1.7-4) as the SVM implementation and set all other parameters to their defaults [37].

\subsubsection{Random Forest}

A random forest $(\mathrm{RF})$ generates multiple decision trees and uses information from each tree to make predictions. This is the best classification model in previous text classification research [38] compared with SVM, Bayes classifier, and k-nearest neighbor algorithm. We used the version package 4.6-14 [39] as the RF implementation and set all the parameters to their default values. 


\subsubsection{Decision Tree}

A decision tree is a non-parametric method among the supervised learning methods. Supervised learning means automatically building predictive models via algorithms from a given set of observations (data) as a training dataset [40]. Test datasets are used to assess how good the algorithm predicts the outcome from unseen data, which is also known as model evaluation. For decision tree analysis, the variables do not need to be linear/normal or additive, and their possible interactions do not need to be pre-specified. Missing values of the covariates, multicollinearity, and outliers are automatically taken into account [41]. We used the party package (R package version 1.3-6) [42] as the decision tree implementation and set all other parameters to their default values.

\subsubsection{Artificial Neural Network}

An Artificial Neural Network is a computational model inspired by the functioning of the human brain. It is composed by a set of artificial neurons (known as processing units) that are interconnected with other neurons. Each connection has an associated weight that represents the influence of one neuron on another. The word network in Neural Network refers to the interconnection between neurons present in various layers of a system. Every system is basically a 3-layered system, and the layers are the Input layer, the Hidden Layer, and the Output Layer. The input layer has input neurons which transfer data via synapses to the hidden layer, and similarly the hidden layer transfers these data to the output layer via more synapses. The synapses store values called weights which help them to manipulate the input and output to various layers. An ANN can be defined based on the following three characteristics:

1. The architecture indicating the number of layers and the number of nodes in each layer.

2. The learning mechanism applied for updating the weights of the connections.

3. The activation functions used in various layers. We used the MXNet version 0.8.0 package [43] to implement the above architecture. The settings used for the training model were as follows: (1) the network architecture was $4 \times 3 \times 1$, i.e., the input layer had 4 nodes, the hidden layer had 3 nodes, and the output layer had 1 node; (2) minibatch gradient descent with batch size of 20 for optimization; (3) learning rate $=0.013$; (4) momentum coefficient $=0.9 ;(4) \mathrm{L} 2$ regularization coefficient $=0$.

\section{Results}

From 2 March 2020 to 4 May 2020, there were 217 cases suspected of COVID-19 who were admitted to our isolation ward at Tri Service General Hospital. The median patient age was 40.8 years (range, 1-92 years). Among them, 107 (49.3\%) were male, 101 (46.5\%) had pneumonia, while $27(12.4 \%)$ were finally confirmed to have contracted COVID-19.

\subsection{Demographic Data and Underlying Diseases of Confirmed COVID-19 Patients and COVID-19-Negative Patients}

The demographic data and underlying diseases of confirmed COVID-19 patients and COVID-19-negative patients are listed in Table 1. There was no gender predominance in both the confirmed group (male vs. female; $51.9 \%$ vs. $48.1 \%$ ) and the negative group (male vs. female; $48.9 \%$ vs. $51.1 \%$ ). The median age of the confirmed patients was $41.7 \pm 18.5$ years, while that of the negative patients was $40.7 \pm 20.4$ years. The confirmed COVID-19 patients had a higher prevalence of hyperlipidemia than the COVID-19-negative patients $(18.5 \%$ vs. $2.6 \% ; p<0.001)$. Between the two groups, there was no significant difference in the prevalence of hypertension, diabetes mellitus, hyperuricemia, chronic kidney disease, cerebrovascular accident, coronary artery disease, cardiac arrhythmia, valvular heart disease, congestive heart failure, bronchial asthma, chronic obstructive pulmonary disease, solid organ cancer, hematogenic disorder, human immunodeficiency virus infection, chronic hepatitis, auto-immune disease, chronic urticaria, or allergic rhinitis 
Table 1. Demographic data and underlying diseases of confirmed COVID-19 patients and COVID-19negative patients.

\begin{tabular}{|c|c|c|c|c|}
\hline & & Confirmed Patients & Negative Patients & \\
\hline \multirow[t]{2}{*}{ Sex } & male & $14(51.9 \%)$ & $93(48.9 \%)$ & $p=0.778$ \\
\hline & female & $13(48.1 \%)$ & $97(51.1 \%)$ & \\
\hline Age (years) & & $41.7 \pm 18.5$ & $40.7 \pm 20.4$ & $p=0.801$ \\
\hline \multicolumn{5}{|c|}{ Underlying diseases } \\
\hline \multirow[t]{2}{*}{ HTN } & yes & $3(11.1 \%)$ & $32(16.8 \%)$ & $p=0.449$ \\
\hline & no & $24(88.9 \%)$ & $158(83.2 \%)$ & \\
\hline \multirow[t]{2}{*}{$\mathrm{DM}$} & yes & $1(3.7 \%)$ & $15(7.9 \%)$ & $p=0.436$ \\
\hline & no & $26(96.3 \%)$ & $175(92.1 \%)$ & \\
\hline \multirow[t]{2}{*}{ Hyperlipidemia } & yes & $5(18.5 \%)$ & $5(2.6 \%)$ & $p<0.001$ \\
\hline & no & $22(81.5 \%)$ & $185(97.4 \%)$ & \\
\hline \multirow[t]{2}{*}{ Hyperuricemia } & yes & $1(3.7 \%)$ & $2(1.1 \%)$ & $p=0.27$ \\
\hline & no & $26(96.3 \%)$ & $188(98.9 \%)$ & \\
\hline \multirow[t]{2}{*}{ CKD } & yes & 0 & $2(1.1 \%)$ & $p=0.592$ \\
\hline & no & $27(100 \%)$ & $188(98.9 \%)$ & \\
\hline \multirow[t]{2}{*}{ CVA } & yes & $1(3.7 \%)$ & $2(1.1 \%)$ & $p=0.27$ \\
\hline & no & $26(96.3 \%)$ & $188(98.9 \%)$ & \\
\hline \multirow[t]{2}{*}{ CAD } & yes & 0 & $7(3.7 \%)$ & $p=0.311$ \\
\hline & no & $27(100 \%)$ & $183(96.3 \%)$ & \\
\hline \multirow[t]{2}{*}{ Cardiac arrhythmia } & yes & 0 & $3(1.6 \%)$ & $p=0.511$ \\
\hline & no & $27(100 \%)$ & $187(98.4 \%)$ & \\
\hline \multirow[t]{2}{*}{ VHD } & yes & 0 & $4(2.1 \%)$ & $p=0.447$ \\
\hline & no & $27(100 \%)$ & $186(97.9 \%)$ & \\
\hline \multirow[t]{2}{*}{$\mathrm{CHF}$} & yes & 0 & $8(4.2 \%)$ & $p=0.447$ \\
\hline & no & $27(100 \%)$ & $182(95.8 \%)$ & \\
\hline \multirow[t]{2}{*}{ Bronchial asthma } & yes & 0 & $7(3.7 \%)$ & $p=0.311$ \\
\hline & no & $27(100 \%)$ & $183(96.3 \%)$ & \\
\hline \multirow[t]{2}{*}{ COPD } & yes & 0 & $2(1.1 \%)$ & $p=0.592$ \\
\hline & no & $27(100 \%)$ & $188(98.9 \%)$ & \\
\hline \multirow[t]{2}{*}{ Solid organ cancer } & yes & $1(3.7 \%)$ & $5(2.6 \%)$ & $p=0.751$ \\
\hline & no & $26(96.3 \%)$ & $185(97.4 \%)$ & \\
\hline \multirow[t]{2}{*}{ Hematogenic disorder } & yes & 0 & $2(1.1 \%)$ & $p=0.592$ \\
\hline & no & $27(100 \%)$ & $188(98.9 \%)$ & \\
\hline \multirow[t]{2}{*}{ HIV infection } & yes & 0 & $2(1.1 \%)$ & $p=0.592$ \\
\hline & no & $27(100 \%)$ & $188(98.9 \%)$ & \\
\hline \multirow[t]{2}{*}{ Chronic hepatitis } & yes & $2(7.4 \%)$ & $5(2.6 \%)$ & $p=0.189$ \\
\hline & no & $25(92.6 \%)$ & $185(97.4 \%)$ & \\
\hline \multirow[t]{2}{*}{ Autoimmune disease } & yes & 0 & $5(2.6 \%)$ & $p=0.394$ \\
\hline & no & $27(100 \%)$ & $185(97.4 \%)$ & \\
\hline \multirow[t]{2}{*}{ Chronic urticaria } & yes & 0 & $3(1.6 \%)$ & $p=0.511$ \\
\hline & no & $27(100 \%)$ & $187(98.4 \%)$ & \\
\hline \multirow[t]{2}{*}{ Allergic rhinitis } & yes & $1(3.7 \%)$ & $2(1.1 \%)$ & $p=0.27$ \\
\hline & no & $26(96.3 \%)$ & $188(98.9 \%)$ & \\
\hline
\end{tabular}

$\mathrm{CAD}$, coronary artery disease; CHF, congestive heart failure; CKD, chronic kidney disease; COPD, chronic obstructive pulmonary disease; COVID-19, coronavirus disease; CVA, cerebrovascular accident; DM, diabetes mellitus; HIV, human immunodeficiency virus; HTN, hypertension; VHD, valvular heart disease.

\subsection{Symptoms of Confirmed COVID-19 and COVID-19-Negative Patients}

The symptoms of confirmed COVID-19 and COVID-19-negative patients are listed in Table 2. The most frequent symptoms in both groups were cough and fever (confirmed cases vs. negative cases; $81.5 \%$ vs. $52.1 \%$ and $63 \%$ vs. $43.7 \%$, respectively). The confirmed COVID-19 patients, compared to the negative patients, had a higher prevalence of cough $(81.5 \%$ vs. $52.1 \% ; p=0.004)$, distorted sense of taste $(25.9 \%$ vs. $0 ; p<0.001)$, distorted sense of smell ( $37 \%$ vs. $0.5 \%$; $p<0.001)$, rhinorrhea ( $44.4 \%$ vs. $14.2 \% ; p<0.001)$, chest tightness $(18.5 \%$ vs. $6.3 \% ; p=0.027)$, dyspnea ( $37 \%$ vs. $12.6 \% ; p=0.001)$, diarrhea ( $33.3 \%$ vs. $5.3 \%$; $p<0.001)$, and nausea and vomiting $(11.1 \%$ vs. $2.1 \%$; $p=0.013)$. On the contrary, there was 
no significant difference in the prevalence of fever, headache, muscle ache, sore throat, or eye illness.

Table 2. Symptoms of confirmed COVID-19 patients and COVID-19-negative patients.

\begin{tabular}{|c|c|c|c|c|}
\hline \multicolumn{2}{|l|}{ Symptoms } & \multirow{2}{*}{$\begin{array}{c}\text { Confirmed Patients } \\
17(63 \%)\end{array}$} & \multirow{2}{*}{$\begin{array}{c}\text { Negative Patients } \\
83(43.7 \%)\end{array}$} & \multirow[b]{2}{*}{$p=0.06$} \\
\hline Fever & yes & & & \\
\hline & no & $10(37 \%)$ & $107(56.3 \%)$ & \\
\hline \multirow[t]{2}{*}{ Cough } & yes & $22(81.5 \%)$ & $99(52.1 \%)$ & $p=0.004$ \\
\hline & no & $5(18.5 \%)$ & $91(47.9 \%)$ & \\
\hline \multirow[t]{2}{*}{ Headache } & yes & $4(14.8 \%)$ & $19(10 \%)$ & $p=0.447$ \\
\hline & no & $23(85.2 \%)$ & $171(90 \%)$ & \\
\hline \multirow[t]{2}{*}{ Muscle ache } & yes & $5(18.5 \%)$ & $15(7.9 \%)$ & $p=0.074$ \\
\hline & no & $22(81.5 \%)$ & $175(92.1 \%)$ & \\
\hline \multirow[t]{2}{*}{ Distorted sense of taste } & yes & $7(25.9 \%)$ & 0 & $p<0.001$ \\
\hline & no & $20(74.1 \%)$ & $190(100 \%)$ & \\
\hline \multirow[t]{2}{*}{ Distorted sense of smell } & yes & $10(37 \%)$ & $1(0.5 \%)$ & $p<0.001$ \\
\hline & no & $17(63 \%)$ & $189(99.5 \%)$ & \\
\hline \multirow[t]{2}{*}{ Rhinorrhea } & yes & $12(44.4 \%)$ & $27(14.2 \%)$ & $p<0.001$ \\
\hline & no & $15(55.6 \%)$ & $163(85.8 \%)$ & \\
\hline \multirow[t]{2}{*}{ Sore throat } & yes & $8(29.6 \%)$ & $32(16.8 \%)$ & $p=0.109$ \\
\hline & no & $19(70.4 \%)$ & $158(83.2 \%)$ & \\
\hline \multirow[t]{2}{*}{ Chest tightness } & yes & $5(18.5 \%)$ & $12(6.3 \%)$ & $p=0.027$ \\
\hline & no & $22(81.5 \%)$ & $178(93.7 \%)$ & \\
\hline \multirow[t]{2}{*}{ Dyspnea } & yes & $10(37 \%)$ & $24(12.6 \%)$ & $p=0.001$ \\
\hline & no & $17(63 \%)$ & $166(87.4 \%)$ & \\
\hline \multirow[t]{2}{*}{ Diarrhea } & yes & $9(33.3 \%)$ & $10(5.3 \%)$ & $p<0.001$ \\
\hline & no & $18(66.7 \%)$ & $180(94.7 \%)$ & \\
\hline \multirow[t]{2}{*}{ Eye illness } & yes & $1(3.7 \%)$ & $1(0.5 \%)$ & $p=0.106$ \\
\hline & no & $26(96.3 \%)$ & $189(99.5 \%)$ & \\
\hline \multirow[t]{2}{*}{ Nausea and vomiting } & yes & $3(11.1 \%)$ & $4(2.1 \%)$ & $p=0.013$ \\
\hline & no & $24(88.9 \%)$ & $186(97.9 \%)$ & \\
\hline
\end{tabular}

COVID-19, coronavirus disease 2019.

\subsection{Laboratory and Radiological Findings of Confirmed COVID-19 Patients and COVID-19-Negative Cases}

The laboratory and radiological findings of confirmed COVID-19 patients and COVID-19negative cases on admissions are listed in Table 3. Confirmed COVID-19 patients had a lower absolute neutrophil count ( $3436.7 \pm 1151.8$ cells $/ \mu \mathrm{L}$ vs. $7011.1 \pm 8888.9$ cells $/ \mu \mathrm{L} ; p=0.038)$ and a lower absolute lymphocyte count $(1334.4 \pm 645.5$ cells $/ \mu \mathrm{L}$ vs. $1912.4 \pm 1357.8$ cells $/ \mu \mathrm{L}$; $p=0.031)$ than COVID-19-negative cases. Among them, 17 (63\%) confirmed COVID-19 patients and 84 (44.2\%) COVID-19-negative patients had pneumonia.

3.4. Accuracy, Sensitivity, and Specificity of Support Vector Machine (SVM), Decision Tree, Random Forest, and Artificial Neural Network for COVID-19 Detection and Diagnosis

The accuracy, sensitivity, and specificity of the AI models we used for COVID-19 detection and diagnosis are shown in Table 4 . In the performance of the models, SVM showed the highest sensitivity (SVM vs. decision tree vs. random forest vs. artificial neural network: $100 \%$ vs. $42.86 \%$ vs. $28.57 \%$ vs. $71.43 \%$ ), while decision tree and random forest had the highest specificity (SVM vs. decision tree vs. random forest vs. artificial neural network: $88.37 \%$ vs. $100 \%$ vs. $100 \%$ vs. $94.74 \%$ ). 
Table 3. Laboratory and radiological findings of confirmed COVID-19 patients and COVID-19negative patients on admission

\begin{tabular}{|c|c|c|c|c|}
\hline & & Confirmed Patients & Negative Patients & \\
\hline \multirow[t]{13}{*}{$\mathrm{Lab}$} & $\mathrm{WBC}(/ \mu \Lambda)$ & $5239 \pm 1498$ & $9907 \pm 13,371$ & $p=0.072$ \\
\hline & Neutrophil (\%) & $65.4 \pm 11.4$ & $68.6 \pm 14.3$ & $p=0.27$ \\
\hline & $\operatorname{ANC}(/ \mu \mathrm{L})$ & $3436.7 \pm 1151.8$ & $7011.1 \pm 8888.9$ & $p=0.038$ \\
\hline & Lymphocyte (\%) & $25.5 \pm 11.1$ & $23 \pm 12.7$ & $p=0.332$ \\
\hline & $\operatorname{ALC}(/ \mu \mathrm{L})$ & $1334.4 \pm 645.5$ & $1912.4 \pm 1357.8$ & $p=0.031$ \\
\hline & $\mathrm{CRP}(\mathrm{mg} / \mathrm{dL})$ & $1.8 \pm 3.1$ & $3.1 \pm 6.1$ & $p=0.117$ \\
\hline & PCT (ng/mL) & $0.08 \pm 0.11$ & $0.55 \pm 0.84$ & $p=0.071$ \\
\hline & D-dimer $(\mathrm{mg} / \mathrm{L})$ & $0.85 \pm 1.8$ & $4.1 \pm 8.1$ & $p=0.089$ \\
\hline & $\operatorname{AST}(\mathrm{U} / \mathrm{L})$ & $21.1 \pm 7.5$ & $26.8 \pm 31.8$ & $p=0.353$ \\
\hline & ALT $(\mathrm{U} / \mathrm{L})$ & $18.6 \pm 8.6$ & $27.4 \pm 37.3$ & $p=0.242$ \\
\hline & Total bilirubin $(\mathrm{mg} / \mathrm{dL})$ & $0.53 \pm 0.24$ & $1.01 \pm 1.50$ & $p=0.2$ \\
\hline & $\mathrm{BUN}(\mathrm{mg} / \mathrm{dL})$ & $13.2 \pm 8.1$ & $13.6 \pm 9.0$ & $p=0.84$ \\
\hline & $\mathrm{Cr}(\mathrm{mg} / \mathrm{dL})$ & $0.82 \pm 0.3$ & $0.96 \pm 1.26$ & $p=0.57$ \\
\hline \multirow[t]{2}{*}{ Pneumonia } & yes & $17(63 \%)$ & $84(44.2 \%)$ & \\
\hline & no & $10(37 \%)$ & $106(55.8 \%)$ & $p=0.068$ \\
\hline
\end{tabular}

AST, aspartate aminotransferase; ALT, alanine aminotransferase; ANC, absolute neutrophil count; ALC, absolute lymphocyte count; BUN, blood urea nitrogen; COVID-19, coronavirus disease 2019; Cr, creatinine; CRP, C-reactive protein; PCT: procalcitonin; WBC, white blood cell.

Table 4. Accuracy, area under the curve (AUC), sensitivity, specificity, positive prediction value (PPV), and negative predictive value (NPV) of support Vector Machine (SVM), decision tree, random forest, and artificial neural network for COVID-19 detection and diagnosis.

\begin{tabular}{ccccccc}
\hline Model & Accuracy & $\begin{array}{c}\text { Area under the } \\
\text { Curve (AUC) }\end{array}$ & Sensitivity & Specificity & $\begin{array}{c}\text { Positive } \\
\text { Prediction } \\
\text { Value (PPV) }\end{array}$ & $\begin{array}{c}\text { Negative } \\
\text { Predictive } \\
\text { Value (NPV) }\end{array}$ \\
\hline Support Vector Machine (SVM) & $88.89 \%$ & $64.29 \%$ & $100.00 \%$ & $88.37 \%$ & $28.57 \%$ & $100 \%$ \\
Decision tree & $91.11 \%$ & $71.43 \%$ & $42.86 \%$ & $100.00 \%$ & $100 \%$ & $90.48 \%$ \\
Random Forest & $88.88 \%$ & $64.29 \%$ & $28.57 \%$ & $100.00 \%$ & $100 \%$ & $88.37 \%$ \\
Artificial Neural Network & $91.11 \%$ & $83.83 \%$ & $71.43 \%$ & $94.74 \%$ & $71.43 \%$ & $94.74 \%$ \\
\hline
\end{tabular}

\section{Discussion}

COVID-19 spread worldwide in just two months since December 2019. Taiwan has been containing it thanks to efforts in early pre-assessment and appraisal to control the disease risk. Between March 22020 and May 4 2020, 217 patients with possible COVID-19 were admitted to our isolation wards, with 27 confirmed cases. These 27 confirmed patients recovered and were discharged, with no occurrence of hospital outbreak.

According to the Taiwan Centers for Disease Control and Prevention (CDC), $934(89.0 \%)$ of the 1050 confirmed COVID-19 cases in Taiwan were imported. Thus, travelers entering Taiwan and symptomatic patients with a travel history are thought to be at the highest risk of SARS-CoV-2 infection. A previous study revealed that the real-time effective reproduction number $(\mathrm{R}(\mathrm{t}))$ of SARS-CoV-2 was 3.27 for Italy, 6.32 for France, 6.07 for Germany, and 5.08 for Spain [5]. With the time-dependent method, the R(t) value was 3.1 for Italy, 6.56 for France, 4.43 for Germany, and 3.95 for Spain [44]. Owing to the highly contagious nature of SARS-CoV-2, people with close contact with confirmed patients are also considered to be at the highest risk. Thus, physicians in our hospital are requested to be aware of high-risk groups and/or individuals, i.e., travelers entering Taiwan, symptomatic patients with a travel or contact history, and people with close contact with confirmed patients, and are requested to follow the well-designed and efficient assessment protocol of COVID-19 screening, hospitalization, and home quarantine presented in Figure 1. The timely identification of individuals at risk may be one of the main factors that assisted Taiwan in containing the pandemic and in preventing outbreaks in its hospitals. 
In our study, confirmed COVID-19 patients had a higher prevalence of hyperlipidemia. According to a New York-based study, the most common comorbidities in COVID-19 fatalities were hypertension (55.4\%), diabetes (37.3\%), hyperlipidemia (18.5\%), and coronary artery disease (12.4\%) [45]. In another study conducted in Wuhan, of the 138 patients hospitalized for COVID-19 and requiring an intensive care support, $25 \%$ had cardiovascular disease, and $58 \%$ had hypertension. Of those who did not require an intensive care units support, $10 \%$ had cardiovascular disease and $22 \%$ had hypertension [46]. According to these studies and our study, dysfunction of lipid metabolism, associated metabolic dysfunction, or related complications such as atherosclerotic disease may increase the vulnerability or severity of COVID-19. Further studies are needed to clarify the relationship between lipid metabolism and COVID-19 pathophysiology.

Similar to several previous studies, we noted cough and fever to be the most common symptoms in confirmed COVID-19 patients [46-49]. In contrast to the what observed for severe acute respiratory syndrome (SARS) of 2003, fever is not considered an important indicator for SARS-CoV-2 transmission. Several studies documented SARS-CoV-2 transmission during the pre-symptomatic incubation period [50-53], while others documented SARS-CoV-2 infection in patients who never developed symptoms (asymptomatic) [54-56]. As shown in an increasing number of studies that report a high prevalence of distorted sense of taste or smell in COVID-19 patients [57,58], 37\% of our confirmed COVID-19 patients had a distorted sense of smell, and $25.9 \%$ had a distorted sense of taste. This could be due to the high expression level of angiotensin-converting enzyme 2 (ACE2) proteins in nasal respiratory epithelial cells and olfactory epithelial support cells [59]. A previous study suggested that the loss of taste and smell, in combination, is a strong predictor of SARS-CoV-2 infection [60]. Physicians should be on alert when patients have distorted smell or taste.

Considering the laboratory findings noted in our study, confirmed COVID-19 patients had a higher prevalence of neutropenia or lymphopenia than COVID-19-negative patients. According to previous studies, lymphopenia was found to be the most common laboratory finding in COVID-19 patients [48,61,62]. Several factors may contribute to COVID-19related lymphopenia. First, lymphocytes express the ACE2 receptor on their surface and may be a direct target of SARS-CoV-2 [63]. Second, the subsequent cytokine storm with increased expression of interleukin-6 (IL-6), granulocyte colony-stimulating factor (GCSF), tumor necrosis factor (TNF)- $\alpha$, and other pro-inflammatory cytokines may cause lymphocyte dysfunction or apoptosis $[64,65]$. Third, the substantial cytokine storm may also be associated with a dysfunction of lymphoid organs such as the spleen [66]. Lymphopenia is considered one of the predictive factors of severe disease in COVID-19 patients [67,68]. Physicians should be vigilant for the presence of lymphopenia in undiagnosed or confirmed COVID-19 patients.

Early detection and timely diagnosis of COVID-19 infections is very helpful to reduce the spread of the virus. However, the nonspecific clinical characteristics of COVID-19 infections make the diagnosis even more difficult. Thus, the development of AI tools for a timely diagnosis of COVID-19 infection is important and imperative, especially in the circumstances when we only have few data regarding suspicious cases. During the pandemic, many AI models were developed for the early detection of COVID-19. Among them, models based on chest computed tomography (CT) images were the most abundant [1-18]. Several studies have developed AI techniques to detect and identify features from chest $\mathrm{CT}$ images to assist in the diagnosis of COVID-19 with high accuracy (70.00 to $99.87 \%$ ), sensitivity (73.00 to $100.00 \%$ ), specificity (25 to $100.00 \%$ ), and AUC (0.732 to 1.000) [1-18]. However, performing CT scan in all suspected COVID-19 patients may cause significant pressure in countries with limited healthcare resources during the pandemic. In our study, we describe a model to early detect COVID-19 infection by inputting clinical characteristics and routine lab data which is more feasible and economic than routine expensive examinations such as chest CT. With the aid of the AI models we 
developed, we can identify patients at risk of COVID-19 and early decide who should be quarantined and undergo further exams such as chest CT or RT-PCR.

However, the present study has several limitations that must be considered. First, we enrolled only 217 suspected COVID-19 patients in our study. The small sample size may affect the reliability of the results because it leads to a higher variability, which may cause bias. Second, the study patients were enrolled almost 2 years ago. The sensitivity and specificity of the AI models we used to identify COVID-19 maybe different when applying to current diagnosed patients. Third, these AI models may help physicians to identify symptomatic COVID-19 patients earlier by analyzing their clinical characteristics. However, asymptomatic patients may not be identified by using our AI systems. Forth, our study was conducted during the COVID-19 pandemic. As we know, behavioral changes (social distancing, mask wearing, and hygiene measures) and travel and movement restrictions during the COVID-19 pandemic have led to a reduction in the incidence of influenza and other common respiratory infections $[69,70]$. The high sensitivity and specificity of the AI models we used to identify COVID-19 maybe due to the reduction in the incidence of other common respiratory infections. Further AI-assisted detection tools still need to be studied and developed.

\section{Conclusions}

In conclusion, COVID-19 is a highly contagious disease characterized by a long period of communicability, varied presentations, and nonspecific laboratory findings. Physicians should be aware of the clinical characteristics of the disease and keenly observe patients at high risk. Besides, the AI-assisted system for the early detection and timely diagnosis of COVID-19 infection needs to be further developed.

Author Contributions: C.-H.C.: designed research. Y.-C.W., Y.-H.Y., T.-T.C., L.-C.Y., T.-Y.L., K.-M.Y.: collected data. Y.-C.W. and D.-J.T.: analyzed data. Y.-C.W. and C.-H.C.: wrote the manuscript. C.-H.C. and F.-Y.C.: revised the entire manuscript. All authors have read and agreed to the published version of the manuscript.

Funding: This work was funded by Tri-Service General Hospital, Taipei, Taiwan [grant numbers TSGH-C108-135, TSGH-C109-147, and 801GB110058] and the National Science Council, Taipei, Taiwan [grant numbers MOST 106-2314-B016-024-MY3, MOST 109-2327-B-016-004].

Institutional Review Board Statement: The study was conducted according to the guidelines of the Declaration of Helsinki and approved by the Institutional Review Board of Tri Service General Hospital (C202005067, 11 May 2020).

Informed Consent Statement: Informed consent was obtained from all subjects involved in the study, and written informed consent was obtained from the patients to publish this paper.

Data Availability Statement: All data generated or analyzed during this study are included in this published article.

Conflicts of Interest: The authors declare no conflict of interest.

\section{References}

1. Bai, H.X.; Wang, R.; Xiong, Z.; Hsieh, B.; Chang, K.; Halsey, K.; Tran, T.M.L.; Choi, J.W.; Wang, D.C.; Shi, L.B.; et al. Artificial intelligence augmentation of radiologist performance in distinguishing COVID-19 from pneumonia of other origin at chest CT. Radiology 2020, 296, E156-E165. [CrossRef] [PubMed]

2. Abbasian Ardakani, A.; Acharya, U.R.; Habibollahi, S.; Mohammadi, A. COVIDiag: A clinical CAD system to diagnose COVID-19 pneumonia based on CT findings. Eur. Radiol. 2020, 31, 121-130. [CrossRef] [PubMed]

3. Ardakani, A.A.; Kanafi, A.R.; Acharya, U.R.; Khadem, N.; Mohammadi, A. Application of deep learning technique to manage COVID-19 in routine clinical practice using CT images: Results of 10 convolutional neural networks. Comput. Biol. Med. 2020, 121, 103795. [CrossRef]

4. Ko, H.; Chung, H.; Kang, W.S.; Kim, K.W.; Shin, Y.; Kang, S.J; Lee, J.H.; Kim, Y.J; Kim, N.Y.; Jung, H.; et al. COVID-19 pneumonia diagnosis using a simple $2 \mathrm{~d}$ deep learning framework with a single chest CT image: Model development and validation. J. Med. Internet Res. 2020, 22, e19569. [CrossRef] [PubMed] 
5. $\quad$ Li, L.; Qin, L.; Xu, Z.; Yin, Y.; Wang, X.; Kong, B.; Bai, J.; Lu, Y.; Fang, Z.; Song, Q.; et al. Using artificial intelligence to detect COVID-19 and community-acquired pneumonia based on pulmonary CT: Evaluation of the diagnostic accuracy. Radiology 2020, 296, E65-E71. [CrossRef]

6. Mei, X.; Lee, H.C.; Diao, K.Y.; Huang, M.; Lin, B.; Liu, C.; Xie, Z.; Ma, Y.; Robson, P.M.; Chung, M.C.; et al. Artificial intelligenceenabled rapid diagnosis of patients with COVID-19. Nat. Med. 2020, 26, 1224-1228. [CrossRef]

7. Mishra, A.K.; Das, S.K.; Roy, P.; Bandyopadhyay, S. Identifying COVID19 from chest CT images: A deep convolutional neural networks based approach. J. Healthc. Eng. 2020, 2020, 8843664. [CrossRef]

8. Ouyang, X.; Huo, J.; Xia, L.; Shan, F.; Liu, J.; Mo, Z.; Yan, F.; Ding, Z.; Yang, Q.; Song, B.; et al. Dual-Sampling attention network for diagnosis of COVID-19 from community acquired pneumonia. IEEE Trans. Med. Imaging 2020, 39, 2595-2605. [CrossRef]

9. Sharma, S. Drawing insights from COVID-19-infected patients using CT scan images and machine learning techniques: A study on 200 patients. Environ. Sci. Pollut. Res. Int. 2020, 27, 37155-37163. [CrossRef]

10. Wang, J.; Bao, Y.; Wen, Y.; Lu, H.; Luo, H.; Xiang, Y.; Li, X.; Liu, C.; Qian, D. Prior-Attention residual learning for more discriminative COVID-19 screening in CT images. IEEE Trans. Med. Imaging 2020, 39, 2572-2583. [CrossRef]

11. Wang, S.; Zha, Y.; Li, W.; Wu, Q.; Li, X.; Niu, M.; Wang, M.; Qiu, X.; Li, H.; Yu, H.; et al. A fully automatic deep learning system for COVID-19 diagnostic and prognostic analysis. Eur. Respir. J. 2020, 56, 2000775. [CrossRef] [PubMed]

12. Wu, X.; Hui, H.; Niu, M.; Li, L.; Wang, L.; He, B.; Yang, X.; Li, L.; Li, H.; Tian, J.; et al. Deep learning-based multi-view fusion model for screening 2019 novel coronavirus pneumonia: A multicentre study. Eur. J. Radiol. 2020, 128, 109041. [CrossRef] [PubMed]

13. Yan, T.; Wong, P.K.; Ren, H.; Wang, H.; Wang, J.; Li, Y. Automatic distinction between COVID-19 and common pneumonia using multi-scale convolutional neural network on chest CT scans. Chaos Solitons Fractals 2020, 140, 110153. [CrossRef] [PubMed]

14. Zhang, K.; Liu, X.; Shen, J.; Li, Z.; Sang, Y.; Wu, X.; Zha, Y.; Liang, W.; Wang, C.; Wang, K.; et al. Clinically applicable AI system for accurate diagnosis, quantitative measurements, and prognosis of COVID-19 pneumonia using computed tomography. Cell 2020, 181, 1423-1433.e11. [CrossRef] [PubMed]

15. Harmon, S.A.; Sanford, T.H.; Xu, S.; Turkbey, E.B.; Roth, H.; Xu, Z.; Yang, D.; Myronenko, A.; Anderson, V.; Amalou, A.; et al Artificial intelligence for the detection of COVID-19 pneumonia on chest CT using multinational datasets. Nat. Commun. 2020, 11, 4080. [CrossRef] [PubMed]

16. Jaiswal, A.; Gianchandani, N.; Singh, D.; Kumar, V.; Kaur, M. Classification of the COVID-19 infected patients using DenseNet201 based deep transfer learning. J. Biomol. Struct. Dyn. 2020, 39, 5682-5689. [CrossRef] [PubMed]

17. Xu, X.; Jiang, X.; Ma, C.; Du, P.; Li, X.; Lv, S.; Yu, L.; Ni, Q.; Chen, Y.; Su, J. A deep learning system to screen novel coronavirus disease 2019 pneumonia. Engineering 2020, 6, 1122-1129. [CrossRef]

18. Yang, S.; Jiang, L.; Cao, Z.; Wang, L.; Cao, J.; Feng, R.; Zhang, Z.; Xue, X.; Shi, Y.; Shan, F. Deep learning for detecting corona virus disease 2019 (COVID-19) on high-resolution computed tomography: A pilot study. Ann. Transl. Med. 2020, 8, 450. [CrossRef]

19. Li, Z.; Zhong, Z.; Li, Y.; Zhang, T.; Gao, L.; Jin, D.; Sun, Y.; Ye, X.; Yu, L.; Hu, Z.; et al. From community-acquired pneumonia to COVID-19: A deep learning-based method for quantitative analysis of COVID-19 on thick-section CT scans. Eur. Radiol. 2020, 30, 6828-6837. [CrossRef]

20. Yu, Z.; Li, X.; Sun, H.; Wang, J.; Zhao, T.; Chen, H.; Ma, Y.; Zhu, S.; Xie, Z. Rapid identification of COVID-19 severity in CT scans through classification of deep features. Biomed. Eng. Online 2020, 19, 63. [CrossRef]

21. Fu, L.; Li, Y.; Cheng, A.; Pang, P.; Shu, Z. A novel machine learning-derived radiomic signature of the whole lung differentiates stable from progressive COVID-19 infection: A retrospective cohort study. J. Thorac. Imaging 2020, 35, 361-368. [CrossRef] [PubMed]

22. Xiao, L.S.; Li, P.; Sun, F.; Zhang, Y.; Xu, C.; Zhu, H.; Cai, F.Q.; He, Y.L.; Zhang, W.F.; Ma, S.C.; et al. Development and validation of a deep learning-based model using computed tomography imaging for predicting disease severity of coronavirus disease 2019 Front. Bioeng. Biotechnol. 2020, 8, 898. [CrossRef] [PubMed]

23. Alsayed, A.; Sadir, H.; Kamil, R.; Sari, H. Prediction of epidemic peak and infected cases for COVID-19 disease in Malaysia, 2020 Int. J. Environ. Res. Public Health 2020, 17, 4076. [CrossRef] [PubMed]

24. Ayyoubzadeh, S.M.; Ayyoubzadeh, S.M.; Zahedi, H.; Ahmadi, M.; Kalhori, S.R.N. Predicting COVID-19 incidence through analysis of google trends data in iran: Data mining and deep learning pilot study. JMIR Public Health Surveill. 2020, 6, e18828. [CrossRef]

25. Mollalo, A.; Rivera, K.M.; Vahedi, B. Artificial neural network modeling of novel coronavirus (COVID-19) incidence rates across the continental United States. Int. J. Environ. Res. Public Health 2020, 17, 4204. [CrossRef]

26. Shahid, F.; Zameer, A.; Muneeb, M. Predictions for COVID-19 with deep learning models of LSTM, GRU and Bi-LSTM. Chaos Solitons Fractals 2020, 140, 110212. [CrossRef]

27. Zheng, N.; Du, S.; Wang, J.; Zhang, H.; Cui, W.; Kang, Z.; Yang, T.; Lou, B.; Chi, Y.; Long, H.; et al. Predicting COVID-19 in China using hybrid AI model. IEEE Trans. Cybern. 2020, 50, 2891-2904. [CrossRef]

28. Arora, P.; Kumar, H.; Panigrahi, B.K. Prediction and analysis of COVID-19 positive cases using deep learning models: A descriptive case study of India. Chaos Solitons Fractals 2020, 139, 110017. [CrossRef]

29. Chimmula, V.K.R.; Zhang, L. Time series forecasting of COVID-19 transmission in Canada using LSTM networks. Chaos Solitons Fractals 2020, 135, 109864. [CrossRef] 
30. Ribeiro, M.; da Silva, R.G.; Mariani, V.C.; Coelho, L.D.S. Short-term forecasting COVID-19 cumulative confirmed cases: Perspectives for Brazil. Chaos Solitons Fractals 2020, 135, 109853. [CrossRef]

31. Shastri, S.; Singh, K.; Kumar, S.; Kour, P.; Mansotra, V. Time series forecasting of Covid-19 using deep learning models: India-USA comparative case study. Chaos Solitons Fractals 2020, 140, 110227. [CrossRef] [PubMed]

32. Gardner, L. Update January 31: Modeling the Spreading Risk of 2019-nCoV. Available online: https://systems.jhu.edu/research/ public-health/ncov-model-2/ (accessed on 31 January 2020).

33. Cheng, S.C.; Chang, Y.C.; Fan Chiang, Y.L.; Chien, Y.C.; Cheng, M.; Yang, C.H.; Huang, C.H.; Hsu, Y.N. First case of Coronavirus Disease 2019 (COVID-19) pneumonia in Taiwan. J. Formos. Med. Assoc. 2020, 119, 747-751. [CrossRef] [PubMed]

34. Wunderink, R.G.; Waterer, G.W. Clinical practice. Community-acquired pneumonia. N. Engl. J. Med. 2014, 370, 543-551. [CrossRef] [PubMed]

35. Postma, D.F.; van Werkhoven, C.H.; van Elden, L.J.; Thijsen, S.F.; Hoepelman, A.I.; Kluytmans, J.A.; Boersma, W.G.; Compaijen, C.J.; van der Wall, E.; Prins, J.M.; et al. Antibiotic treatment strategies for community-acquired pneumonia in adults. N. Engl. J. Med. 2015, 372, 1312-1323. [CrossRef] [PubMed]

36. Butt, L.; Zuccon, G.; Nguyen, A.; Bergheim, A.; Grayson, N. Classification of cancer-related death certificates using machine learning. Australas. Med. J. 2013, 6, 292-299. [CrossRef]

37. Meyer, D.; Dimitriadou, E.; Hornik, K.; Weingessel, A.; Leisch, F. Misc Functions of the Department of Statistics, Probability Theory Group (Formerly: E1071), R package version 1.7-0; Vienna University of Technology (TU Wien): Vienna, Austria, 2018.

38. Mohammed Ali Al-garadi, K.D.V.; Ravana, S.D. Cybercrime detection in online communications: The experimental case of cyberbullying detection in the Twitter network. Comput. Hum. Behav. 2016, 63, 433-443. [CrossRef]

39. Liaw, A.; Wiener, M. Classification and regression based on a forest of trees using random inputs, based on Breiman (2001). $R$ Doc. Package Randomforest 2018, 4, 14.

40. Geurts, P.; Irrthum, A.; Wehenkel, L. Supervised learning with decision tree-based methods in computational and systems biology. Mol. Biosyst. 2009, 5, 1593-1605. [CrossRef]

41. Speybroeck, N.; Berkvens, D.; Mfoukou-Ntsakala, A.; Aerts, M.; Hens, N.; van Huylenbroeck, G.; Thysa, E. Classification trees versus multinomial models in the analysis of urban farming systems in Central Africa. Agric. Syst. 2004, 80, 133-149. [CrossRef]

42. Hothorn, T.; Hornik, K.; Zeileis, A. Unbiased Recursive Partitioning: A Conditional Inference Framework. J. Comput. Graph. Stat. 2006, 15, 651-674. [CrossRef]

43. Chen, T.; Li, M.; Li, Y.; Lin, M.; Wang, N.; Wang, M.; Xiao, T.; Xu, B.; Zhang, C.; Zhang, Z. Mxnet: A flexible and efficient machine learning library for heterogeneous distributed systems. arXiv 2015, arXiv:preprint/1512.01274.

44. Yuan, J.; Li, M.; Lv, G.; Lu, Z.K. Monitoring transmissibility and mortality of COVID-19 in Europe. Int. J. Infect. Dis. 2020, 95, 311-315. [CrossRef]

45. Saldanha, A.L.R.; Chacra, A.P.M.; Rabelo, L.M.; Filho, R.D.S.; Margeotto, A.P.P.; Gasparoto, A.L.V.; Martinez, T.L.D.R. COVID 19 comorbidity interplayers diabetes and atherogenic dyslipidemia. Curr. Trends Intern. Med. 2020, 4, 138.

46. Wang, D.; Hu, B.; Hu, C.; Zhu, F.; Liu, X.; Zhang, J.; Wang, B.; Xiang, H.; Cheng, Z.; Xiong, Y.; et al. Clinical Characteristics of 138 Hospitalized Patients With 2019 Novel Coronavirus-Infected Pneumonia in Wuhan, China. JAMA 2020, 323, 1061-1069. [CrossRef] [PubMed]

47. Chen, N.; Zhou, M.; Dong, X.; Qu, J.; Gong, F.; Han, Y.; Qiu, Y.; Wang, J.; Liu, Y.; Wei, Y.; et al. Epidemiological and clinical characteristics of 99 cases of 2019 novel coronavirus pneumonia in Wuhan, China: A descriptive study. Lancet 2020, 395, 507-513. [CrossRef]

48. Huang, C.; Wang, Y.; Li, X.; Ren, L.; Zhao, J.; Hu, Y.; Zhang, L.; Fan, G.; Xu, J.; Gu, X.; et al. Clinical features of patients infected with 2019 novel coronavirus in Wuhan, China. Lancet 2020, 395, 497-506. [CrossRef]

49. Lai, C.C.; Shih, T.P.; Ko, W.C.; Tang, H.J.; Hsueh, P.R. Severe acute respiratory syndrome coronavirus 2 (SARS-CoV-2) and coronavirus disease-2019 (COVID-19): The epidemic and the challenges. Int. J. Antimicrob. Agents 2020, 55, 105924. [CrossRef]

50. Hu, Z.; Song, C.; Xu, C.; Jin, G.; Chen, Y.; Xu, X.; Ma, H.; Chen, W.; Lin, Y.; Zheng, Y.; et al. Clinical characteristics of 24 asymptomatic infections with COVID-19 screened among close contacts in Nanjing, China. Sci. China Life Sci. 2020, 63, 706-711. [CrossRef]

51. Wei, W.E.; Li, Z.; Chiew, C.J.; Yong, S.E.; Toh, M.P.; Lee, V.J. Presymptomatic Transmission of SARS-CoV-2-Singapore, January 23-March 16, 2020. Morb. Mortal. Wkly. Rep. 2020, 69, 411-415. [CrossRef]

52. Tong, Z.D.; Tang, A.; Li, K.F.; Li, P.; Wang, H.L.; Yi, J.P.; Zhang, Y.L.; Yan, J.B. Potential Presymptomatic Transmission of SARS-CoV-2, Zhejiang Province, China, 2020. Emerg. Infect. Dis 2020, 26, 1052-1054. [CrossRef]

53. Qian, G.; Yang, N.; Ma, A.H.Y.; Wang, L.; Li, G.; Chen, X.; Chen, X. COVID-19 Transmission Within a Family Cluster by Presymptomatic Carriers in China. Clin. Infect. Dis. 2020, 71, 861-862. [CrossRef]

54. Pan, X.; Chen, D.; Xia, Y.; Wu, X.; Li, T.; Ou, X.; Zhou, L.; Liu, J. Asymptomatic cases in a family cluster with SARS-CoV-2 infection. Lancet Infect. Dis. 2020, 20, 410-411. [CrossRef]

55. Bai, Y.; Yao, L.; Wei, T.; Tian, F.; Jin, D.Y.; Chen, L.; Wang, M. Presumed Asymptomatic Carrier Transmission of COVID-19. JAMA 2020, 323, 1406-1407. [CrossRef] [PubMed]

56. Rothe, C.; Schunk, M.; Sothmann, P.; Bretzel, G.; Froeschl, G.; Wallrauch, C.; Zimmer, T.; Thiel, V.; Janke, C.; Guggemos, W.; et al Transmission of 2019-nCoV Infection from an Asymptomatic Contact in Germany. N. Engl. J. Med. 2020, 382, 970-971. [CrossRef] [PubMed] 
57. Hopkins, C.; Surda, P.; Whitehead, E.; Kumar, B.N. Early recovery following new onset anosmia during the COVID-19 pandemicAn observational cohort study. J. Otolaryngol.-Head Neck Surg. 2020, 49, 26. [CrossRef]

58. Lee, Y.; Min, P.; Lee, S.; Kim, S.W. Prevalence and Duration of Acute Loss of Smell or Taste in COVID-19 Patients. J. Korean Med. Sci. 2020, 35, e174. [CrossRef]

59. Brann, D.H.; Tsukahara, T.; Weinreb, C.; Lipovsek, M.; Van den Berge, K.; Gong, B.; Chance, R.; Macaulay, I.C.; Chou, H.J.; Fletcher, R.B.; et al. Non-neuronal expression of SARS-CoV-2 entry genes in the olfactory system suggests mechanisms underlying COVID-19-associated anosmia. Sci. Adv. 2020, 6, 31, eabc5801. [CrossRef]

60. Menni, C.; Valdes, A.M.; Freidin, M.B.; Sudre, C.H.; Nguyen, L.H.; Drew, D.A.; Ganesh, S.; Varsavsky, T.; Cardoso, M.J.; Moustafa, J.S.E.; et al. Real-time tracking of self-reported symptoms to predict potential COVID-19. Nat. Med. 2020, 26, 1037-1040. [CrossRef]

61. Guan, W.J.; Ni, Z.Y.; Hu, Y.; Liang, W.H.; Ou, C.Q.; He, J.X.; Liu, L.; Shan, H.; Lei, C.L.; Hui, D.S.C.; et al. Clinical Characteristics of Coronavirus Disease 2019 in China. N. Engl. J. Med. 2020, 382, 1708-1720. [CrossRef]

62. Terpos, E.; Ntanasis-Stathopoulos, I.; Elalamy, I.; Kastritis, E.; Sergentanis, T.N.; Politou, M.; Psaltopoulou, T.; Gerotziafas, G.; Dimopoulos, M.A. Hematological findings and complications of COVID-19. Am. J. Hematol. 2020, 95, 834-847. [CrossRef]

63. Xu, H.; Zhong, L.; Deng, J.; Peng, J.; Dan, H.; Zeng, X.; Li, T.; Chen, Q. High expression of ACE2 receptor of 2019-nCoV on the epithelial cells of oral mucosa. Int. J. Oral. Sci. 2020, 12, 8. [CrossRef] [PubMed]

64. Liao, Y.C.; Liang, W.G.; Chen, F.W.; Hsu, J.H.; Yang, J.J.; Chang, M.S. IL-19 induces production of IL-6 and TNF-alpha and results in cell apoptosis through TNF-alpha. J. Immunol. 2002, 169, 4288-4297. [CrossRef] [PubMed]

65. Aggarwal, S.; Gollapudi, S.; Gupta, S. Increased TNF-alpha-induced apoptosis in lymphocytes from aged humans: Changes in TNF-alpha receptor expression and activation of caspases. J. Immunol. 1999, 162, 2154-2161. [PubMed]

66. Chan, J.F.; Zhang, A.J.; Yuan, S.; Poon, V.K.; Chan, C.C.; Lee, A.C.; Chan, W.M.; Fan, Z.; Tsoi, H.W.; Wen, L.; et al. Simulation of the Clinical and Pathological Manifestations of Coronavirus Disease 2019 (COVID-19) in a Golden Syrian Hamster Model: Implications for Disease Pathogenesis and Transmissibility. Clin. Infect. Dis. 2020, 71, 2428-2446. [CrossRef] [PubMed]

67. Tan, L.; Wang, Q.; Zhang, D.; Ding, J.; Huang, Q.; Tang, Y.Q.; Wang, Q.; Miao, H. Lymphopenia predicts disease severity of COVID-19: A descriptive and predictive study. Signal Transduct. Target. Ther. 2020, 5, 33. [CrossRef] [PubMed]

68. Zhao, Q.; Meng, M.; Kumar, R.; Wu, Y.; Huang, J.; Deng, Y.; Weng, Z.; Yang, L. Lymphopenia is associated with severe coronavirus disease 2019 (COVID-19) infections: A systemic review and meta-analysis. Int. J. Infect. Dis. 2020, 96, 131-135. [CrossRef]

69. Laurie, K.L.; Rockman, S. Which influenza viruses will emerge following the SARS-CoV-2 pandemic? Influenza Other Respir Viruses 2021, 15, 573-576. [CrossRef]

70. Huang, Q.S.; Wood, T.; Jelley, L.; Jennings, T.; Jefferies, S.; Daniells, K.; Nesdale, A.; Dowell, T.; Turner, N.; Campbell-Stokes, P.; et al. Impact of the COVID-19 nonpharmaceutical interventions on influenza and other respiratory viral infections in New Zealand. Nat. Commun. 2021, 12, 1001. [CrossRef] 